\title{
Questes
}

Revue pluridisciplinaire d'études médiévales

\section{L'épisode de la Forêt Perdue dans le Lancelot en prose : jeux et divertissements périlleux en terre de Bretagne}

Patrick Moran

\section{(2) OpenEdition}

\section{Journals}

Édition électronique

URL : http://journals.openedition.org/questes/671

DOI : $10.4000 /$ questes.671

ISSN : 2109-9472

Éditeur

Les Amis de Questes

\section{Édition imprimée}

Date de publication : 15 février 2010

Pagination : 87-102

ISSN : 2102-7188

\section{Référence électronique}

Patrick Moran, «L'épisode de la Forêt Perdue dans le Lancelot en prose : jeux et divertissements périlleux en terre de Bretagne », Questes [En ligne], 18 | 2010, mis en ligne le 01 janvier 2014, consulté le 03 mai 2019. URL : http://journals.openedition.org/questes/671 ; DOI : 10.4000/questes.671 


\section{L'épisode de la Forêt Perdue dans le Lancelot en prose : jeux et divertissements périlleux en terre de Bretagne}

Patrick MORAN

L'épisode de la Forêt Perdue (ou "de la carole magique " en référence à l'un de ses éléments surnaturels) appartient au dernier tiers du

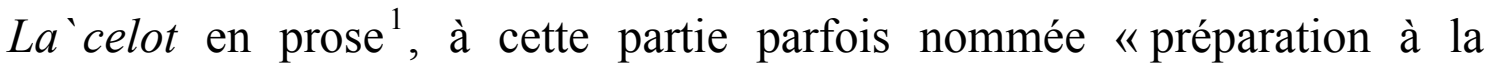
Queste », où les aventures du héros et des autres chevaliers de la Table Ronde s'éloignent de plus en plus du terreau courtois des débuts et s'orientent vers l'horizon mystique du Graal. Dans ce contexte, l'aventure que rencontre Lancelot au cœur de la Forêt Perdue offre un interlude léger, marqué par une thématique à la fois amoureuse et ludique; bien que l'épisode en lui-même soit d'une longueur textuelle limitée, sa construction singulière et les ramifications qui en découlent méritent que l'on s'y attarde. En mettant en scène deux jeux enchantés - une danse et un jeu d'échecs l'aventure crée un réseau signifiant qui interroge, à travers des activités de divertissement, leurs équivalents « sérieux », l'amour et la guerre, et mettent en lumière ce qu'il y a de réglé, et de déréglé, dans l'univers chevaleresque.

L'épisode débute lorsque Lancelot, accompagné d'un écuyer, pénètre dans une forêt et découvre un avertissement menaçant écrit sur un perron :

Si erra en tel maniere dusqu'a none qu'il entra en .I. forest vielle et ancienne. Il voit a l'antree une chapele ou il avoit .I. hermite molt prodome, et tout entor la chapele avoit .I. cimetire et a l'entree .I. croiz et .I. grant perron de marbre. Il esgarde sor le perron lestres vermeilles qui disoient: «Os tu, chevaliers erranz qui ceste part

\footnotetext{
${ }^{1}$ Volumes IV, V et VI de l'édition d'Alexandre Micha (La 'celot, Alexandre MichA (éd.), Paris/Genève, Droz, 1978-1983).
} 
viens por aventure trover, se tu ne vels morir, n'entrer mie en ceste forest, car tu n'am porroies eschaper sanz mort ou sanz honte. $\rangle^{2}$

L'ermite explique aux deux voyageurs qu'il s'agit de la Forêt Perdue, dont nul n'est jamais revenu, et ce depuis bien des années. Il leur déconseille vivement de s'y rendre. Lancelot passe la nuit chez l'ermite et repart en direction de la forêt le lendemain avec son écuyer, qui est de plus en plus réticent. Dans les bois ils croisent une demoiselle qui leur adresse un avertissement similaire, tout aussi inquiétant.

Peu après ils parviennent à une clairière où se trouvent une tour, un trône d'ivoire sur lequel est posée une couronne, trente tentes et trois pins autour desquels dansent des chevaliers et des demoiselles en "caroles ", c'est-à-dire en rondes joyeuses. Lancelot est rassuré de voir un spectacle aussi gai au cœur d'une forêt aussi sinistre, mais il est aussitôt pris au piège et entre dans la ronde, qui est enchantée :

Et quant Lanceloz vint cele part et il vit les queroles dont li pin estoient avironné, si s'en merveille molt et toutes voies dist au vallet: « Molt a ci bele compaingnie et ranvoisie gent ! Il ne monstrent mie que l'an ne puist par ceste forest aler seurement et dahaiz aie, se je ne vois savoir de quoi il font si grant feste. » Lors se fiert es pavillons et si tost com il a le premier encontré, si li mue li sans et li change li talanz : car s'il devant n'avoit talant fors de chevalerie et d'assaut et de meslees comancier, or est ses voloirs a ce menez qu'il n'a talant fors de queroler; si oublie sa dame et ses compaingnons et soi meesmes en tel manniere qu'il ne l'an souvient mais, ainz descent de son cheval et le baille a garder au vallet, si giete sa lance et son escu a terre et s'en vait a la querole toz armez, le hiaume lacié, et se prent a la premiere damoisele qu'il encontre. Et lors conmance a chanter et a ferir del pié ausi conme li autre, si s'anvoise et joue assez plus qu'il n'avoit onques mes fet et tant que li vallez meesmes le resgarda et le tient por fol. Et il chantoient une chançon qui estoit faite de la roine Genievre et il chantoient en escotois si que li vallez n'antandoit mie bien qu'il disoient et neporquant tant en savoit que li sans de la parole estoit tiex: "Voirement avons nos la plus bele roine des autres. $»^{3}$

\footnotetext{
${ }^{2}$ La celot IV, p. 229-230.

${ }^{3}$ La`celot IV, p. 234-235.
} 
L'écuyer tente de s'adresser à Lancelot, mais celui-ci lui ordonne de le laisser danser. Le valet attend jusqu'au coucher du soleil. Il essaie à nouveau de faire partir Lancelot, mais le chevalier l'ignore; il chante comme les autres une nouvelle chanson, «Voirement fait il bon maintenir amors ». L'écuyer, comprenant qu'il s'agit d'un enchantement, abandonne Lancelot et s'en va. À ce moment le récit de l'épisode s'interrompt, et cède la place à des aventures d'Yvain, de Bohort et de Gauvain : le texte met ainsi en scène de façon saisissante, par le silence, le temps passé à danser, un temps fait de vide et de répétition. Ce n'est que bien plus tard que l'entrelacement revient à l'épisode de la Forêt Perdue, où Lancelot est toujours dans la carole ${ }^{4}$. Vient l'heure du souper: une des demoiselles demande à Lancelot de s'asseoir sur le trône et d'essayer la couronne ; il obtempère, et aussitôt les enchantements cessent :

Quant il fu tans de souper, si vint une damoisele a lui qui li dist: "Sire chevaliers, il vos estuet aler seoir en cele chaiere, si vos mestrons cele couronne d'or en vostre teste. » Et il dist qu'il n'a cure de couronne ne de chaiere ne ne velt riens fors joie et anvoiseure. « Il couvient, fait ele, que vos i ailliez, car par ce connoistrions nos se nos devons estre delivré par vos. Et se vos ne nos delivrez, il estuet que vos remaingniez ci o nos et atandrons tant que Diex nos amaint celui par cui nos devons estre delivré de ceste folie ou nos somes. » Et il dist qu'il i ira volentiers, puis qu'ele le velt : si se vait aseoir en la chaiere et cele li mest la couronne en la teste et li dist : "Biax sire, or poez dire que vos avez la couronne vostre pere en vostre teste. » Et il se resgarde et voit chaoir de la tor en haut une ymage qui ert faite en samblance de roi et ert entaillie moult richement et flati a la terre si durement qu'ele fu toute desquassee. Et maintenant failli li anchantemenz, si revindrent tuit en lor sans et en lor mimoire dont il avoient esté longuement soufraiteux ${ }^{5}$.

Un vieux chevalier explique l'origine de la carole : peu de jours après le mariage d'Arthur et de Guenièvre, le roi Ban de Bénoïc, père de

\footnotetext{
${ }^{4}$ La première partie de l'épisode s'étend, dans l'édition Micha, des pages 229 à 236 ; la deuxième partie, des pages 286 à 297.

${ }^{5}$ La celot IV, p. 286.
} 
Lancelot, se promenait dans cette forêt avec sa suite et un cousin qui était clerc et enchanteur ${ }^{6}$. Ils rencontrent un groupe de demoiselles qui dansent, avec à leur tête une magnifique jeune femme assise sur un trône. Ban encourage ses hommes à danser avec les demoiselles, et celle assise sur le trône émet le vœu que de telles réjouissances durent toujours. Le clerc, qui est tombé amoureux d'elle, la prend au mot et jette un enchantement sur la clairière : toute personne qui entre dedans, et qui aime ou a un jour aimé, doit être éternellement entraînée dans la ronde. Seul le meilleur et le plus beau chevalier du monde pourra rompre l'enchantement. Le roi Ban, avant de repartir, dépose sa couronne sur le trône et proclame que celui qui brisera l'enchantement aura mérité de la porter. Le clerc et la demoiselle restent sur place pour vivre leur amour. Au début la demoiselle est heureuse, mais au bout de quatorze ans elle s'ennuie et demande qu'il lui fasse un nouveau jeu : il lui offre donc un échiquier magique qui joue tout seul et qui est imbattable. Un jour, annonce-t-il, viendra un chevalier « gracieuz et desirrez et amez sor touz autres et cil savra tant des eschés et d'autres jeus qu'il ne trovera son pareil el monde de soutillesce et par celui seront maté cil eschés $»^{7}$.

Lancelot, entendant cette histoire, demande évidemment à jouer contre l'échiquier, et remporte la partie. Avant de quitter la Forêt Perdue, il envoie un des chevaliers avec le jeu d'échecs auprès de la reine Guenièvre pour le lui offrir. Arthur et Guenièvre reçoivent le jeu mais sont incapables

\footnotetext{
${ }^{6}$ Dans la Suite Vulgate du Merli', l'épisode de la création de la carole est mis en scène, et le cousin anonyme devient un personnage plus fouillé, sous le nom de Guinebaut. La version de la Suite Vulgate manifeste une différence de taille avec celle du La`celot: la carole magique est conçue pour emprisonner tout le monde et non seulement les personnes qui connaissent ou ont connu l'amour, et elle est vouée à être vaincue non pas par le plus valeureux et le plus beau chevalier au monde, mais par un amant qui n'aurait jamais été infidèle - contamination manifeste par l'épisode du Val des Faux Amants (cf. i fra, note 10). Le sens de l'épreuve s'en trouve profondément changé. Voir Le Livre du Graal, Philippe WALter (éd.), Paris, Gallimard, 3 vol., 2001-2009; tome 1, p. 11391144.

${ }^{7}$ La celot IV, p. 292.
} 
de le battre, bien que la reine soit réputée être la meilleure joueuse du royaume. Le récit cesse alors de parler de la Forêt Perdue, mais y revient sous forme de corollaire, après de nombreuses aventures : Lancelot revient finalement à Kamaalot, et Arthur lui demande d'affronter l'échiquier ; Lancelot le bat à nouveau et s'attire l'admiration de tous, et tout particulièrement du roi $^{8}$.

L'épisode de la Forêt Perdue intervient à un moment inattendu dans l'organisation du La 'celot en prose. Son contenu en soi n'est pas inhabituel, et la combinaison d'une coutume magique, d'un contexte courtois et d'une épreuve qualifiante fonde bon nombre d'aventures du roman. Mais cet épisode, on l'a dit, survient assez tard dans le récit, dans un contexte qui a évolué. La jeunesse du héros a cédé le pas à la maturité, et le style des aventures a changé en conséquence, d'autant plus que la Queste del sai 't Graal se profile de plus en plus à l'horizon du texte : le discours religieux s'intensifie aux dépens du discours courtois. L'aventure de la carole magique suit même de peu une visite de Lancelot au château du Graal, pendant laquelle il assiste au cortège du Graal et conçoit Galaad à son insu avec la fille du Roi Pêcheur - épisode-clé dans l'économie cyclique s'il en est, en fort contraste par sa tonalité mystique avec celui qui le suit. S'il fallait rapprocher la Forêt Perdue d'autres aventures, ce serait plutôt des épreuves qui émaillent les deux premiers tiers du roman, où Lancelot doit délivrer une population emprisonnée par enchantement: on songe aux épisodes de la Douloureuse Garde ${ }^{9}$, d'Escalon le Ténébreux ${ }^{10}$, du Val des Faux Amants ${ }^{11}$ ou de la terre de Gorre ${ }^{12}$. De toutes ces épreuves, c'est celle du Val des Faux Amants que la Forêt Perdue évoque le plus : créé par

\footnotetext{
${ }^{8}$ Cet épilogue occupe, dans l'édition Micha, les pages 392-393.

${ }^{9}$ La celot VII, p. 311-333.

${ }^{10}$ La'celot I, p. 254-266.

${ }^{11}$ La'celot I, p. 266-304.

${ }^{12}$ La`celot II, p. 1-108.
} 
Morgane, ce val est un piège qui attire les hommes qui ont été infidèles à leur dame, sans espoir d'en ressortir un jour à moins qu'un amant parfait échappe à l'enchantement et le brise. La Forêt Perdue offre en quelque sorte une vision inversée de cette aventure : là où Lancelot était l'exception qui confirmait la règle dans l'épisode du Val, le seul amant parfaitement fidèle, il est ici le parangon des vertus que célèbre la carole magique. Les qualités qui lui permettaient d'échapper au piège de Morgane sont précisément celles qui provoquent son emprisonnement dans la Forêt. La similarité en miroir de ces deux épisodes est renforcée par les noms de lieux : l'ermite dit de la Forêt Perdue qu'elle est « la Voie sans retour », de même que le Val des Faux Amants, également appelé «Val sans retour ».

\section{Des jeux dangereux}

L'épisode ressemble aussi à d'autres aventures, où Lancelot est confronté à une double épreuve; habituellement il n'est voué à en accomplir qu'une seule des deux, soit parce qu'il vient (dans les débuts du roman) compléter ce qu'un autre chevalier n'a pu réussir en entier ${ }^{13}$, soit (et c'est plus souvent le cas dans la «préparation à la Queste ») parce qu'un autre chevalier, plus noble et plus pur que lui, viendra terminer ce qu'il a commencé $^{14}$. La Forêt Perdue est peut-être le seul cas où Lancelot est doublement le chevalier élu, celui qui était destiné par ses actes ou par ses qualités à vaincre tous les enchantements - même si ces mêmes qualités sont aussi celles qui l'ont rendu captif.

\footnotetext{
${ }^{13}$ Cf. par exemple l'épisode de la maladie d'Agravain, qui débute dans La celot VIII, p. 226-235. Agravain, pour être soigné, doit être oint du sang des deux meilleurs chevaliers au monde; le meilleur doit lui oindre la jambe et le deuxième le bras. Gauvain ne parvient à lui guérir que le bras : seul Lancelot, un peu plus tard, pourra guérir sa jambe (p. 431-432).

${ }^{14}$ Il s'agit, bien sûr de Galaad. Cf. notamment l'épisode des tombes du Saint Cimetière, La'celot II, p. 1-38.
} 
Ces deux enchantements semblent de nature similaire: le texte emploie le terme de jeu pour les désigner; il les met explicitement en relation lorsque la demoiselle demande au cousin du roi Ban de lui faire un deuxième $«$ jeu $»^{15}$ parce qu'elle se lasse de la carole. La plasticité du terme recouvre pourtant deux activités qui peuvent sembler fort différentes : si les échecs correspondent manifestement à ce que l'anglais nomme game, représentant même le game par excellence dans la culture occidentale, la carole en revanche relève plutôt du domaine du play, le divertissement sans compétition ni vainqueur. Dans la typologie de Roger Caillois, les échecs appartiennent à la catégorie de l'agô', de l'affrontement, tandis que la danse entre dans celle du vertige d'origine sacrée, l'ili $x^{16}$. Un gouffre semble séparer ces deux activités, l'une parfaitement réglée et polie, l'autre débridée et irrationnelle - la nature « civilisée » de la carole étant battue en brèche par son caractère hypnotique et inépuisable.

Ces différences ne doivent pourtant pas faire oublier qu'aux yeux des personnages et du texte, ces deux enchantements appartiennent à une même sphère, et la terminologie le souligne. Ce sont des jeux, et surtout ce sont des jeux de cour: jeux échecs et caroles sont nombreux dans le Cycle Vulgate et la littérature arthurienne en général, et représentent les divertissements raffinés auxquels peuvent s'adonner le roi et les siens. Le choix de ces deux «jeux» permet, dans le cadre d'un seul épisode, de couvrir le spectre des activités arthuriennes qui permettent la rencontre d'égal à égal entre les deux sexes : la danse et le jeu de plateau valorisent la grâce et l'intelligence et non la force physique, et offrent un contexte

${ }^{15}$ La 'celot IV, p. 291 : « Or vos pri je dont, fait ele, si com vos m'amez, que vos .I. autre jeu façoiz a quoi nos nos puissons esbatre ».

${ }^{16}$ Roger CAILlois, Les Jeux et les hommes. Le masque et le vertige, Paris, Gallimard, 1958, p. 25 sqq. : «Classification des jeux ». La définition que donne Caillois de l'ili` $x$ est particulièrement adaptée à la scène de la carole magique : "Une dernière espèce de jeux rassemble ceux qui reposent sur la poursuite $d u$ vertige [...]. Dans tous les cas, il s'agit d'accéder à une sorte de spasme, de tra'se ou d'étourdisseme 't qui a éa 'tit la réalité avec u’e souverai e brusquerie. » (p. 45, c’est nous qui soulignons). 
socialement acceptable où hommes et femmes interagissent sur un pied d'égalité.

Qui dit égalité ne dit pas indifférenciation, car les deux activités véhiculent des connotations courtoises et amoureuses plus ou moins explicites. Dans le cas de la carole magique, la coloration est évidente : hommes et femmes se tiennent la main et célèbrent l'amour par leurs chansons. La carole a été instaurée par le cousin du roi Ban par amour, à l'occasion de l'union d'Arthur et de Guenièvre. La première chanson décrite dans le récit loue la beauté de la reine, tandis que la deuxième est une célébration générale de l'amour. Guenièvre, pourtant absente de la scène, en est un point focal : c'est son mariage avec Arthur qui est à l'origine de l'enchantement, et c'est son amour pour Lancelot qui piègera celui-ci. Ce double rôle involontaire donne à la première chanson une teinte légèrement ironique.

Les connotations amoureuses de l'échiquier sont moins évidentes, mais elles héritent d'une longue tradition intertextuelle; en littérature courtoise le jeu d'échecs est rarement dénué d'une métaphore amoureuse. Le fait que Guenièvre soit la meilleure joueuse de Bretagne n'est là aussi pas anodin : l'égalité des sexes s'en trouve soulignée, et le personnage de la reine se retrouve à nouveau au centre du réseau signifiant de l'épisode. L'échiquier magique a pour particularité non seulement d'être imbattable, mais de toujours mater ses adversaires «en l'angle». L'expression est ambivalente: elle souligne l'excellence de l'échiquier (le «mat en l'angle » étant un coup particulièrement apprécié), mais elle est aussi lourde de sous-entendus : dans de nombreux textes, particulièrement les textes allégoriques, la formule «mater en l'angle» peut avoir un sens courtois - la victoire amoureuse - voire sexuel. Si le narrateur ne joue pas explicitement ici sur l'un ou l'autre double sens, il n'en reste pas moins que 
l'insistance du texte sur la formule, qui est répétée plusieurs fois, est lourde de connotations ${ }^{17}$ pour le lecteur médiéval.

Tous ces éléments contribuent à donner à l'épisode une atmosphère légère, qu'on dirait même " galante » si l'on ne craignait l'anachronisme. Il est même tentant d'y voir un interlude humoristique, lorsqu'on compare les avertissements terribles que reçoit Lancelot et la réalité de l'aventure, et qu'on ajoute à cela la figure incongrue d'un Lancelot, personnage souvent taciturne et solitaire, en train de chanter et de danser avec insouciance. Le La celot en général n'est pas dénué d'humour, mais l'épisode semble revêtir ici une dimension presque parodique, qui n'est pas typique de ce roman. L'enchantement lui-même est sans gravité : inventé comme un divertissement contre l'ennui, il fait office de parenthèse enchantée qui fait oublier tous les soucis et ne fait de mal à personne. Les critères qui permettent de bannir l'enchantement mettent l'accent sur les qualités les plus « superficielles » de Lancelot : c'est le meilleur chevalier, mais surtout le plus beau qui pourra mettre fin à la carole magique. Comme l'explique le cousin du roi Ban, ce qui a commencé par la beauté se terminera par la beauté. Quant au jeu d'échecs, Lancelot le bat parce qu'il est « gracieuz et desirrez et amez sor touz autres », mais aussi parce qu'il est le meilleur joueur d'échecs au monde. Ce sont ses qualités sociales qui lui permettent de l'emporter. Il est entendu que toutes ces qualités - la beauté, la grâce, le caractère plaisant et la compétence au jeu - en dissimulent d'autres, plus sérieuses, et que seul un individu d'exception serait capable de les réunir,

\footnotetext{
${ }^{17}$ Voir à ce sujet l'article de Françoise GuICHARD-TESSON, « Jeux de l'amour et jeux du langage ", Le Moye 'Fra 'çais, 38 (1997), p. 21-44. Le sens sexuel de l'expression est rare en littérature arthurienne, mais on le trouve dans un passage du Livre d'Artus (tome 7 de The Vulgate Versio ' of the Arthuria' Roma 'ces, Heinrich Oscar Sommer (éd.), Washington, The Carnegie Institution, 7 vol., 1908-1916, p. 191), où Sagremor et une demoiselle qu'il vient de délivrer passent la nuit à «mater en l'angle » dans l'intimité d'une chambre : «Molt menerent grande feste entre Sagremor et la damoisele et molt se deduirent longuement, tant qu'il virent le jour aparoir, et commencerent le jeu trois foiz et materent en l'angle. » L'acception, si elle n'est pas fréquente, n'est donc pas impossible dans le contexte d'un roman arthurien en prose.
} 
un être aussi bon que beau, aussi courageux que gracieux ; il n'en reste pas moins que le texte s'évertue à célébrer le versant le plus «frivole» ou mondain des vertus chevaleresques et courtoises.

Simultanément, cette apparente légèreté est battue en brèche par la nature des deux jeux auxquels est confronté Lancelot. Le jeu, si l'on se réfère à la définition de Caillois, est une activité libre, séparée, incertaine, improductive, réglée et fictive ${ }^{18}$. Or l'échiquier propose une activité qui n'est en rien $i$ 'certai ' $e$ : au contraire l'issue en est certaine, qu'on s'appelle ou non Lancelot, puisque dans un cas on perdra à tous les coups et dans l'autre on gagnera en permanence. La carole, elle, n'est pas une activité libre, puisqu'elle fonctionne à peu de choses près comme une prison dorée. Deux traits essentiels qui garantissent le plaisir ludique sont absents. Quant aux autres, ils sont exacerbés : la carole est si séparée du monde habituel et réglée qu'elle fonctionne en vase clos, comme un mécanisme d'horlogerie qui ne servirait à rien.

\section{Les ambiguïtés d'une épreuve qualifiante}

Si l'épisode est singulier par son contenu et par les traits qu'il met en valeur, il l'est peut-être encore plus par sa position dans le roman. Il s'agit d'un interlude courtois et léger dans une partie du La'celot connue pour être plus religieuse et plus grave; le récit fait suivre un moment particulièrement signifiant dans l'économie du cycle - la conception de Galaad à Corbenic - par une aventure qui semble délibérément insignifiante. L'épisode de la Forêt Perdue correspond à ce qu'Annie Combes appelle dans Les Voies de l'ave 'ture une aventure-élection ${ }^{19}$,

\footnotetext{
${ }^{18}$ Roger CAILlOIS, Les Jeux et les hommes. Le masque et le vertige, op. cit., p. 23-24.

${ }^{19}$ Annie Combes, Les Voies de l'ave ture. Réécriture et compositio 'roma esque da 's le Lancelot $e^{\prime}$ prose, Paris, Champion, 2001, p. 391-393. Le terme apparaît dans une typologie des différents types d'aventure chevaleresque, et forme une sous-catégorie de l'« aventure-épreuve».
} 
c'est-à-dire une épreuve que seul le héros peut remporter, en raison de qualités qu'il possède à un point plus haut que nul autre. Mais, point discordant, on a affaire ici à une élection qui se fait sur des critères moins profonds que d'habitude : la beauté vaut plus que la valeur guerrière ou le courage, et autant que l'habileté à jouer aux échecs. Surtout, les aventuresélections se déroulent plutôt dans les premiers temps du La celot, au cours des années de formation du jeune chevalier; l'épisode de la Forêt Perdue arrive fort tard, à un moment où Lancelot n'a plus rien à prouver et a depuis longtemps démontré qu'il était le meilleur chevalier au monde.

$\mathrm{Ou}$ plutôt, l'aventure survient à un moment du roman où la précellence de Lancelot commence à être mise en doute : il n'accomplira pas les aventures du Graal, puisque sa luxure et son adultère avec Guenièvre le condamnent aux yeux de Dieu. La "préparation à la Queste » commence à mettre en place le binôme chevalerie terrie ' $e$-chevalerie celestielle qui fondera le système de valeurs de la Queste del sai`t Graal: Lancelot n'était que le meilleur des chevaliers terriens, il sera bientôt surpassé par un héros d'un autre ordre de grandeur. Dans ce contexte, l'épisode de la carole propose le dernier moment d'exaltation du personnage de Lancelot: il vainc les deux aventures et non une seule, prouve qu'il est le meilleur chevalier et le plus beau, et se fait même coiffer de la couronne de son père ${ }^{20}$. Pourtant, la carole l'a piégé tout comme les autres, et ce en raison de son amour pour Guenièvre, amour qui auparavant lui permettait de vaincre toutes les épreuves. Sa passion pour la reine, moteur de tous ses exploits dans le $L a^{`}$ 'celot, devient un poids qui l'entraîne

\footnotetext{
${ }^{20} \mathrm{Ce}$ détail revêt toute son importance lorsqu'on se souvient qu'à aucun moment Lancelot n'accepte de monter sur le trône de Bénoïc, et se considère jusqu'à la fin du Cycle Vulgate comme un vassal d'Arthur et non comme un souverain. Lancelot, d'ailleurs, retire l'objet très vite après en avoir été couronné; néanmoins la scène permet au récit de lui accorder son statut légitime de roi, ne serait-ce qu'un instant, et de le faire symboliquement monter au faîte de sa gloire.
} 
loin du salut dans la Queste: la carole magique, par son ambivalence, représente un moment pivot dans cette évolution axiologique.

Si l'aventure de la Forêt Perdue marque un point de bascule dans le système de valeurs du La`celot, ou du moins une étape dans son inévitable évolution, elle provoque aussi une mutation dans les relations qu'entretient Lancelot avec les autres. C'est la scène finale à Kamaalot qui permet de mesurer ce changement, lorsque le héros rentre à la cour et fait montre de sa prouesse aux échecs. L'admiration d'Arthur est alors à son comble pour Lancelot, mais cet amour provoque la haine chez un grand nombre de membres de la Table Ronde, qui en secret deviennent aigris et envieux. C'est aussi pendant cette scène de cour que Guenièvre, en écoutant le récit des aventures de son amant, se rend compte que leur amour adultère empêche définitivement Lancelot d'être le chevalier parfait ${ }^{21}$. Cette découverte n'est pas directement liée à l'aventure de la Forêt Perdue, mais le fait qu'elle se fasse en même temps que les retombées de cet épisode est significatif: Lancelot est simultanément à l'acmé de sa gloire terrestre (manifestée par l'admiration d'Arthur), plus détesté qu'il ne l'a jamais été, et condamné à une humiliation spirituelle qui se réalisera dans la Queste. Cette scène de cour, en mettant l'accent aussi bien sur la détresse spirituelle de Lancelot, sur l'amour que lui porte Arthur, et sur sa relation avec Guenièvre et l'inimitié des autres chevaliers, porte en germe non seulement les bouleversements de la Queste del sai't Graal mais aussi ceux de la Mort le roi Artu, où Arthur et Lancelot deviendront ennemis jurés et diviseront la chevalerie bretonne en deux: les ombres portées de cet épisode apparemment léger s'étendent plus loin qu'il n'y paraît.

Dans cette logique évolutive, les deux jeux de la Forêt Perdue permettent à l'idéal courtois de s'exprimer une dernière fois complètement, mais soulignent aussi ses limites : de même que l'amour mène les danseurs

${ }^{21}$ La`celot V, p. 3. 
à faire d'interminables rondes, de même il conduit Lancelot à tourner en rond, prisonnier de son amour charnel et incapable de s'en libérer. Seule une cause supérieure, le Graal, permettra aux chevaliers de Bretagne de sortir de cette ornière ${ }^{22}$. L'égarement de Lancelot est souligné de façon saisissante par le dispositif textuel lui-même: l'entrelacement mime le temps passé par le héros dans un état d'absence total, hypnotisé par la carole magique. Un tel montage textuel dément le caractère apparemment léger de l'aventure et lui donne une résonance particulière : au lieu d'être réglé en une fois, l'épisode scande la lecture et devient plus marquant.

La nature des deux épreuves qualifiantes que doit remporter Lancelot incite à réfléchir aux points communs qu'il y a entre le jeu et l'aventure chevaleresque. Toute aventure n'est-elle pas une sorte de jeu, avec des règles plus ou moins complexes? Un enchantement peut être brisé sous certaines conditions; une coutume exige des chevaliers qu'ils s'y soumettent ou qu'ils essaient de la vaincre. Le monde de l'aventure est, en somme, réglé jusque dans ses dérèglements.

L'errance elle-même s'apparente au jeu lorsqu'elle ne se fixe pas un but supérieur: elle est, comme le jeu chez Caillois, une activité libre, séparée, incertaine, improductive, réglée - et fictive, du moins de notre point de vue de lecteur. Même si le rédacteur du La celot n'a pas lu Caillois, il met en œuvre dans cet épisode une mécanique d'affaiblissement de l'errance chevaleresque «classique », qui se montre sous un jour positif et ludique mais dévoile ses limites. Lancelot a prouvé tout ce qu'il pouvait prouver, et son amour est désormais un frein autant qu'un moteur. L'intérêt

\footnotetext{
${ }^{22}$ Mihaela VoICU, dans son article « La Déconstruction de l'espace dans le 'LancelotGraal' ou vers une nouvelle mesure du monde chevaleresque » (in La Chevalerie $d u$ Moye`Âge à 'os jours. Méla'ges offerts à Michel Sta esco, Mihaela VoICU et VictorDinu Vladulescu (éds.), Bucarest, Editura Universităţii din Bucareşti, 2003, p. 249270), rapproche ainsi la carole magique de la Roue de Fortune dans La Mort le roi Artu, interprétant les deux motifs comme un «mouvement vide autour de l'Être ».
} 
principal de l'épisode de la Forêt Perdue, en dehors de ses détails amusants, vient sans doute de ce croisement de deux discours, de deux régimes narratifs et idéologiques, qui font de cette section du roman un point de bascule décisif où la « règle du jeu » change définitivement.

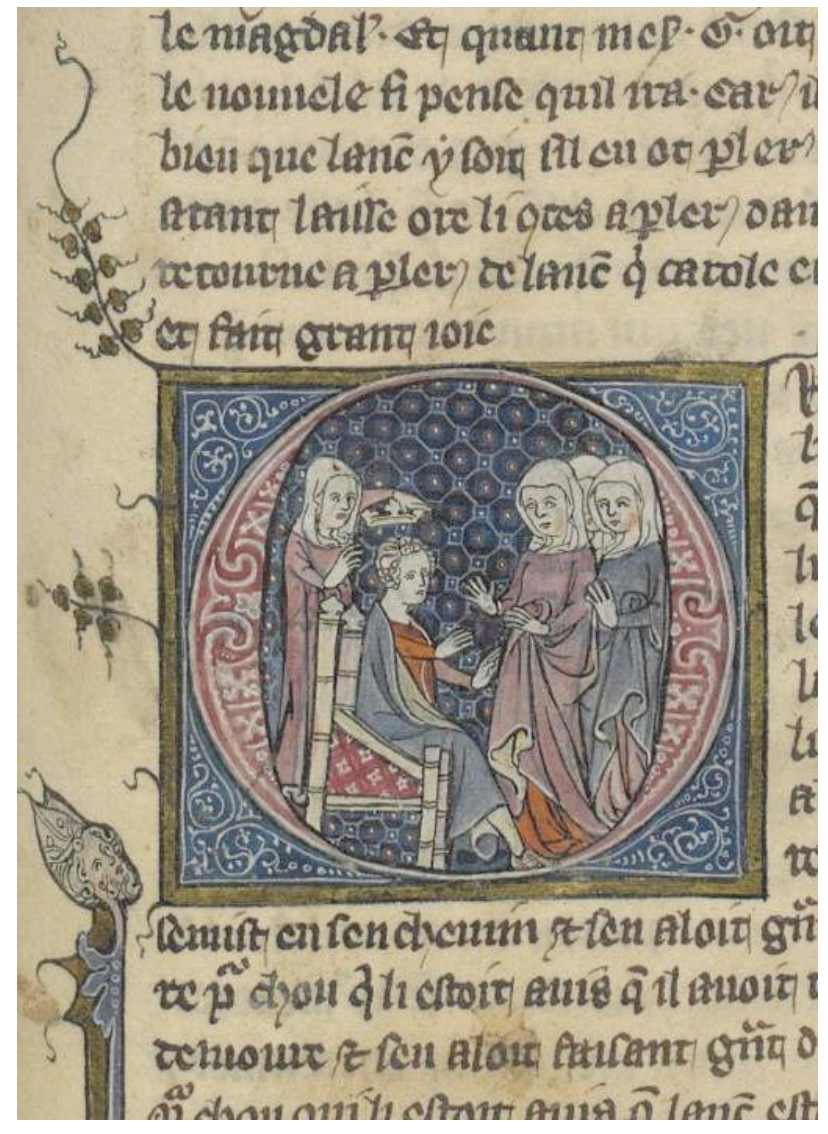

BnF fr. 1422, fo 67 : couronnement de Lancelot. 


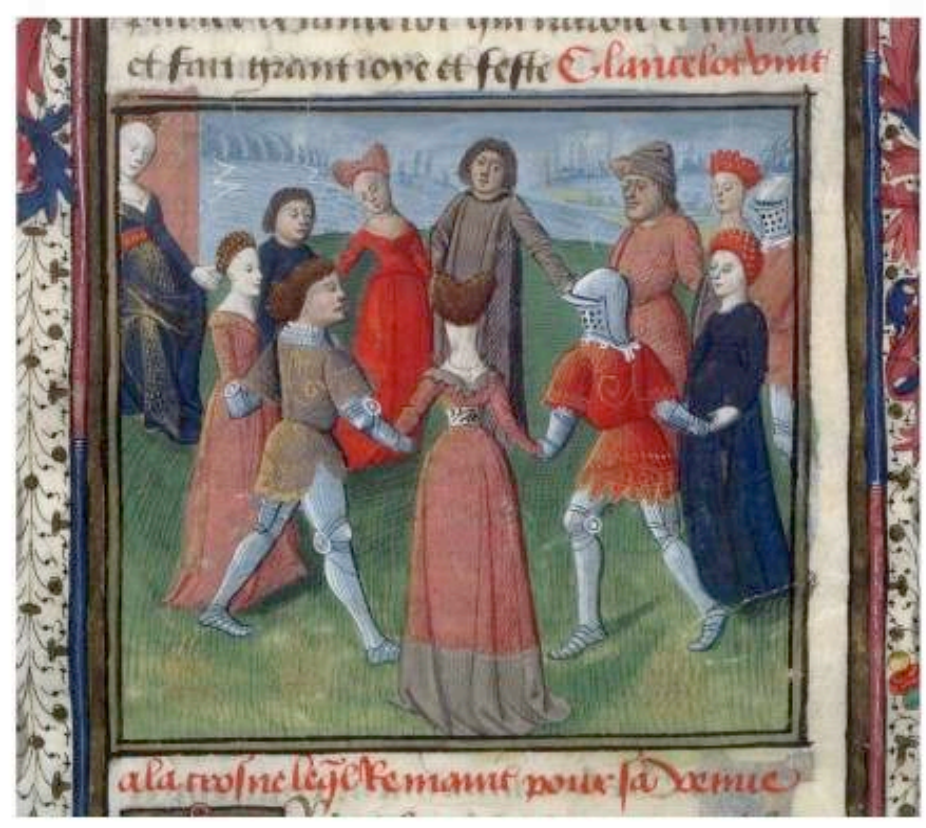

BnF fr. $115, f^{\circ} 476$ : la carole magique.

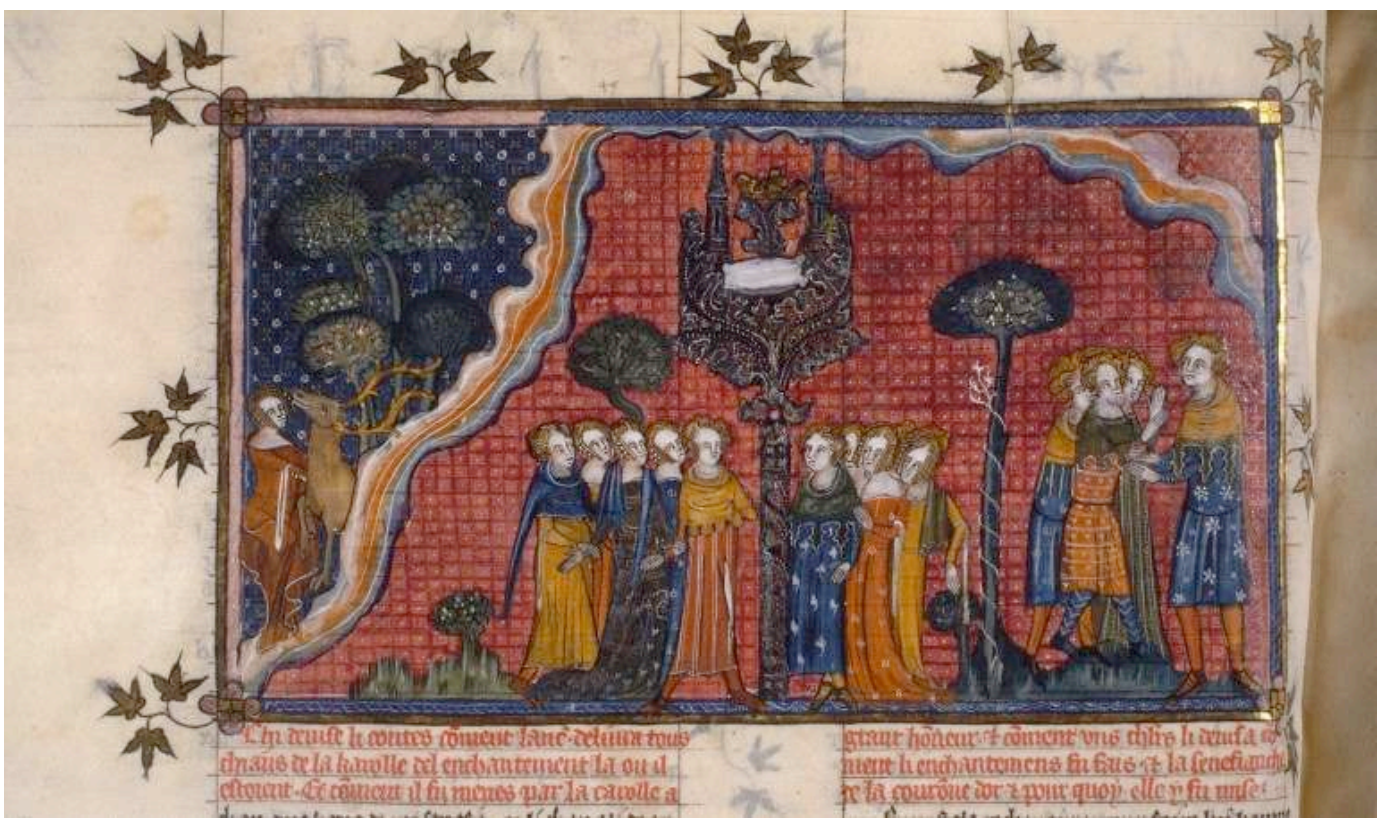

BnF fr. $122, f^{\circ} 137 v^{\circ}$ : la carole magique. 


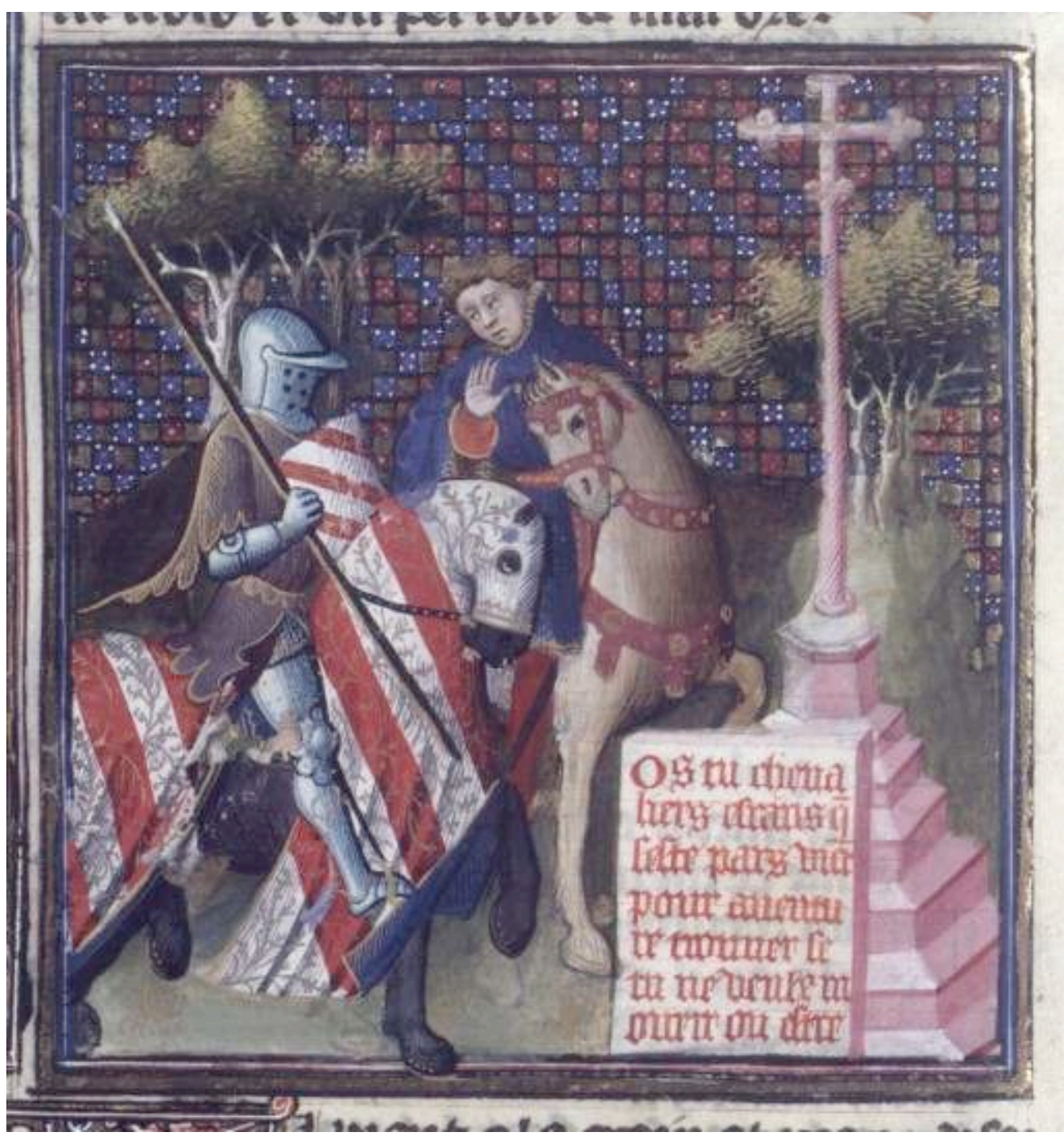

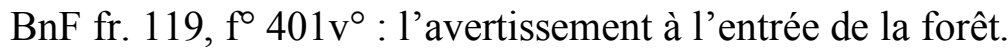

